\title{
STUDY XXIII. THE RELATION BETWEEN AMYLASE RETENTION AND EXCRETION AND NON-PROTEIN NITROGEN RETENTION IN EXPERIMENTAL URANIUM NEPHRITIS *
}

\author{
R. FITZ, M.D. \\ BOSTON
}

Tests for renal function fall into three general groups depending on: (1) The ability of the kidney to excrete from the circulation abnormal substances, such as dyes or drugs which are introduced into it; (2) analysis of the products of metabolism retained in the blood as a result of faulty eliminative powers of the kidney; (3) abnormalities in the physical and chemical properties of the urine.

The phenolsulphonephthalein test is an excellent example of the first group. Rowntree and Geraghty ${ }^{2}$ have found that the amount of this dye excreted in the urine after its intramuscular or intravenous injection varies almost in proportion to the degree of injury existing in the kidneys. This test is used so generally by clinical and experimental observers that its value in the study of the renal function can be accepted without further comment.

Of substances studied in the blood of nephritics the concentration of the non-protein nitrogen and urea is of most importance. Among others Ascoli, ${ }^{2}$ Strauss, ${ }^{3}$ Obermayer and Popper, ${ }^{4}$ Widal ${ }^{5}$ and Foster ${ }^{6}$

* Submitted for publication Sept. 29, 1914.

* From the Laboratory of the Theory and Practice of Physic, Harvard Medical School, and the Medical Clinic of the Peter Bent Brigham Hospital.

* This is one of a series of studies on experimental cardiorenal disease: Study I, Smith: Boston Med. and Surg. Jour., 1908, clviii, 696; Study II, Christian: Boston Med. and Surg. Jour., 1908, clix, 8; Study III, Christian: Jour. Am. Med. Assn., 1909, liii, 1792; Studies IV-XV, Christian, Smith and Walker: The Archives Int. Med., 1911, viii, 468-551; Study XVI, Christian and O'Hare: The Archives Int. Med., 1913, xi, 517; Studies XVII and XVIII, O'Hare: The Archives Int. Med., 1913, xii, 49, 61 ; Study XIX, Christian and O'Hare: Jour. Med. Research, 1913, xxviii, 22\% ; Study XX, Walker and Dawson: Thr Archives Int. Mrd, 1913, xii, 171; Study XXI, Fitz: The Archives Int. Med., 1914, xiii, 945; Study XXII, Christian: 'The Archives Int. Med., 1914, xiv, 827.

1. Rowntree and Geraghty: Jour. Pharm. and Exper. Therap., 1910, i, 579; The Archives Int. Med., 1912, ix, 284.

2. Ascoli: Pflüger's Arch. f. d. ges. Physiol., 1901, 1xxxvii, 103.

3. Strauss: Die Chronische Nierentzündungen in ihrer Einwirkung auf die Blutflüssigkeit und deren Behandlung., Hirschwald, Berlin, 1902.

4. Obermayer and Popper: Ztschr. f. klin. Med., 1909, 1xvii, 332.

5. Widal: Bull. et mém. Soc. méd. d. hôp., Paris, Series 3, 1911, xxxii, 627.

6. Foster: The Archives Int. Med., 1912, x, 414. 
showed that in the majority of cases the nitrogen of the blood increases with an increasingly severe nephritis, and that the degree of accumulated blood nitrogen affords valuable information in regard to the prognosis.

Heretofore, urinalysis, except in relation to the output of chlorids, nitrogen and water, has contributed little to our understanding of renal function. In 1908, however, Wohlgemuth ${ }^{7}$ began a series of studies with the starch-splitting ferment amylase (or diastase). He found that the amount of this enzyme which was excreted in the twenty-four hour urine of normal men varied within narrow limits and was almost constant for a given individual. In the urine of nephritics, on the other hand, the amount of the enzyme excreted in the urine diminished in proportion to the severity of the disease. Wohlgemuth, therefore, suggested that the determination of amylase in urine afforded a valuable clinical test for renal function.

His results with this test have been confirmed both by experiments and by clinical studies. Hirata ${ }^{8}$ showed that in rabbits, as well as in man, the twenty-four hour urine and the blood serum contained normally a constant amount of amylase. When corrosive sublimate, uranium nitrate, or chromic acirl nephritis was produced the amylase in the urine diminished, and accumulated in the blood in almost direct proportion to the severity of the existing lesions. Von Benczur, ${ }^{9}$ Wynhausen, ${ }^{10}$ Rosenthal, ${ }^{11}$ Marino, ${ }^{12}$ Corbett $^{13}$ and Geyelin ${ }^{14}$ have observed the excretion of amylase in nephritis and have found that it diminishes as the severity of the disease increases. Therefore there is sufficient evidence to prove that of substances constantly found in urine the amylase excretion represents a reasonably accurate test for the renal function.

The desirability of correlating as many tests for renal function as possible to determine their true values has been self-evident and has been attempted both in animal experiments and clinical studies. Frothingham, Fitz, Folin and Denis ${ }^{15}$ produced uranium nephritis in rabbits and followed the relation between the non-protein nitrogen retention in the blood and the excretion of phenolsulphonephthalein in the urine. The two tests paralleled each other as indicators of

7. Wohlgemuth: Ueber eine neue Methode zur quantitativen Bestimmung des diastatischen Ferments.

8. Hirata: Biochem. Ztschr., 1910, xxviii, 23.

9. Von Benczur: Wien. klin. Wchnschr., 1910, xxiii, 890.

10. Wynhausen: Berl. klin. Wchnschr., 1910, xlvii, 2107.

11. Rosenthal: Deutsch. med. Wchnschr., 1911, xxxvii, 923.

12. Marino: Deutsch. Arch. f. klin. Med., 1911, ciii, 325.

13. Corbett: Quart. Jour. Med., 1913, vi, 351.

14. Geyelin: The Archives Inr. Med., 1914, xiii, 96.

15. Frothingham, Fitz, Folin and Denis: The Archives Int. Med., 1913, xii, 245. 
renal function, but had one essential difference. The phenolsulphonephthalein excretion in the urine dropped rapidly to its lowest point and returned rapidly to normal with recovery of the kidney. The nonprotein nitrogen of the blood accumulated more gradually and returned to normal more gradually. In other words, the phenolsulphonethalein test showed the renal function at the time the test was made, while the blood nitrogen was rather a measure of an accumulating difference between the amount of waste nitrogen produced in metabolism and the amount eliminated by the kidneys. Therefore in the latter test the duration of the disease was an important factor. Confirmatory results illustrating the close parallelism of these two tests have been obtained in clinical observations by Agnew ${ }^{16}$ and by Frothingham and Smillie. ${ }^{17}$

Geyelin $^{14}$ compared the excretion of amylase with that of phenolsulphonephthalein in a series of cases of nephritis and found that the results of these tests were essentially similar. Geraghty, Rowntree and $\mathrm{Cary}^{18}$ found that although the phenolsulphonephthalein test gave more accurate information as to the degree of one-sided lesions of the kidney than the amylase test, yet the latter gave findings which were parallel.

Thus it has been shown that on the whole the excretion of amylase varies as does that of phenolsulphonephthalein in nephritis, and that as the excretion of phenolsulphonephthalein diminishes, the non-protein nitrogen of the blood accumulates. It seemed important to compare the amylase excretion in the urine and the amylase accumulation in blood serum with the accumulation of non-protein nitrogen in the blood of animals with nephritis to make comparative studies between these three tests more complete.

Accordingly, acute nephritis was produced in rabbits by means of a single dose of uranium nitrate (from 2 to $10 \mathrm{mg}$.) given subcutaneously. Two series of experiments were made. In the first series the animals were injected with uranium nitrate and were killed (under anesthesia) by bleeding from the carotid arteries. They were killed on consecutive days from one to ten days after the uranium nitrate was administered in order to secure histological record of the pathological lesions in the kidney, and to determine their relation to the non-protein nitrogen in the blood, to the amylase in the blood, and to the amylase in the urine at different stages of the nephritis. In these experiments the amylase of the urine was estimated from day to day. The blood was analyzed only on the day the experiment was begun and on the day the animals were killed. The kidney tissues were preserved for

16. Agnew : The Archives Int. Med., 1914, xiii, 485.

17. Frothingham and Smillie: The Archives Int. Med., 1914, xiv, 541.

18. Geraghty, Rowntree and Cary: Ann. Surg., 1913, lviii, 800. 
the histological examination in Zenker's fluid and were stained with eosin and methylene blue.

In the second series of experiments, the nephritis was allowed to run its natural course. The non-protein nitrogen of the blood and the amylase of the urine were determined periodically. Amylase in the blood serum could not be estimated so often on account of the anemia induced from such frequent and relatively large bleedings.

The rabbits were kept in metabolism cages of moderate size. They were fed with carrots, oats and water. Each rabbit consumed about $100 \mathrm{gm}$. of carrot and $50 \mathrm{gm}$. of oats per day except for a few days at the height of the nephritis. In addition each rabbit was given 50 c.c. of water by stomach tube every morning to insure as constant an output of urine as possible.

The urine specimens from the metabolism cages were collected in receivers which contained 10 c.c. of toluol as a preservative. Every morning, the animals' bladders were emptied by massage and the urines so obtained were added to the containers to complete the twentyfour hour specimens.

The amylase readings in both urine and blood serum were made by the method of Wohlgemuth and Noguchi. ${ }^{19}$ In brief, this depends on determining the amount of starch which is converted to dextrin or lower products by a known amount of urine or blood at a given temperature during a given interval of time. Iodin is used as an indicator of the starch digestion. Wohlgemuth has taken for a unit which he calls " $d$ " the number of cubic centimeters of .1 per cent. soluble starch solution digested in one-half hour by 1 c.c. of urine or blood serum at a temperature of $38 \mathrm{C}$. " $\mathrm{D}$ " is the number of cubic centimeters of starch solution digested by the twenty-four hour urine. Rosenthal in obtaining the "absolute diastatic strength" of urine has preferred to divide Wohlgemuth's "D" reading by 2 .

In the experiments with urine both " $\mathrm{d}$ " readings and the "absolute diastatic strength" were recorded to show the effect of dilution on the excretion of amylase. This question has been discussed by various observers. Wynhausen, ${ }^{10}$ Corbett, ${ }^{13}$ Rosenthal, ${ }^{11}$ Von Benczur ${ }^{9}$ and Geyelin" found that the influence of dilution on " $d$ " readings was limited and without effect in the range of normal twenty-four hour urines, although allowance must be made when the quantities were abnormally large or small. In the experiments reported, such marked variations in urinary output occurred from day to day that the necessity of considering the effect of dilution was obvious.

19. Wolgemuth and Noguchi: Berl. klin. Wchnschr., 1912, xlix, 1096. This method is described in detail by Geyelin (Note 14) and therefore the description is not repeated here. 
In the experiments with blood, 5 c.c. were withdrawn and defibrinated by shaking with beads. The serum was obtained by centrifugalization, and its "d" reading made by the method mentioned. Folin and Denis' ${ }^{\prime 20}$ micromethod on oxalated blood was used for the blood nitrogen determinations.

\section{EXPERIMENTS}

Experiment 1.-Rabbit killed on the first day after the administration of the uranium nitrate.
First day. (Wt. $1,650 \mathrm{gm}$.)
Urine: twenty-four-hour amount............... 150 c.c.

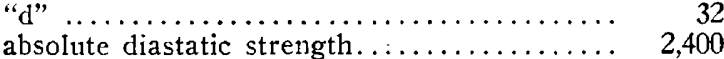
Blood: serum "d" . ....................... 32
non-protein nitrogen (per $100 \mathrm{gm}$.) ........ $37 \mathrm{mg}$.
Administered $3 \mathrm{mg}$. ùranium nitrate.
Second day. (Wt. $1,700 \mathrm{gm}$.)
Urine: twenty-four-hour amount ............. 150 c.c.
"d" $\ldots \ldots \ldots \ldots \ldots \ldots \ldots \ldots \ldots \ldots \ldots \ldots \ldots \ldots \ldots \ldots, \quad 32$
absolute diastatic strength.............. 2,400
Blood: serum "d" ........................... 32
non-protein nitrogen (per $100 \mathrm{gm}$.)........ 54

Histological findings: Glomeruli negative; tubules negative. No pathological changes found in the kidney.

Expermont 2.-Rabbit killed on the second day after the administration of the uranium nitrate.

First day. (Wt. 1,600 gm.)

Urine: twenty-four-hour amount $\ldots \ldots \ldots \ldots \ldots \ldots, \quad 150$ c.c. "d" $\ldots \ldots \ldots \ldots \ldots \ldots \ldots \ldots \ldots \ldots \ldots \ldots . \ldots \ldots, \quad 32$

absolute diastatic strength................ 2,400

Blood: serum "d" ........................ 64 non-protein nitrogen (per $100 \mathrm{gm}.) \ldots \ldots \ldots .25 \mathrm{mg}$.

Administered $6 \mathrm{mg}$. uranium nitrate.

Second day. (Wt. 1,700 gm.)

Urine: twenty-four-hour amount $\ldots \ldots \ldots \ldots \ldots \ldots, 180$ c.c.

"d" ............................... 32

absolute diastatic strength............. 2,880

Third day. (Wt. 1,700 gm.)

Urine: twenty-four-hour amount ............ 150 c.c.

"d" ............................ 64

absolute diastatic strength................ 4,800

Blood: serum "d" ........................ 64 non-protein nitrogen (per $100 \mathrm{gm}$.)........ $44 \mathrm{mg}$.

Histological Findings: Glomeruli negative; slight necrosis and desquamation of the epithelium of certain tubules; a few tubules filled with necrotic material; a few areas of round cell infiltration.

Experiment 3.-Rabbit killed on the third day after the administration of the uranium nitrate.

First day. (Wt. 2,600 gm.)

Urine: twenty-four-hour amount $\ldots \ldots \ldots \ldots \ldots \ldots, 150$ c.c.

"d" .............................. 128

absolute diastatic strength $\ldots \ldots \ldots \ldots \ldots \ldots, \quad 9,600$

Blood: serum "d" ....................... 64 non-protein nitrogen (per $100 \mathrm{gm}.) \ldots \ldots \ldots \ldots .637 \mathrm{mg}$.

Administered $6 \mathrm{mg}$. uranium nitrate.

20. Folin and Denis: Jour. Biol. Chem., 1912, xi, 257. 
Second day. (Wt. 2,600 gm.)

Urine : twenty-four-hour amount $\ldots \ldots \ldots \ldots \ldots \ldots$.

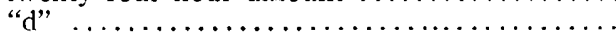
absolute diastatic strength

160 c.c.

Third day. (Wt. 2,500 gm.)

Urine: twenty-four-hour amount $\ldots \ldots \ldots \ldots \ldots \ldots$

$$
\text { "d" }
$$

absolute diastatic strength

Fourth day. (Wt. 2,450 gm.)

Urine: twenty-four-hour amount $\ldots \ldots \ldots \ldots \ldots \ldots$

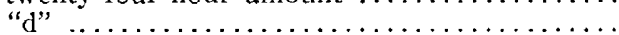
absolute diastatic strength ................

Blood : serum "d" .......................... 128 non-protein nitrogen (per $100 \mathrm{gm}$.)........ $131 \mathrm{mg}$.

Histological Findings: Glomeruli negative. Considerable necrosis and desquamation of tubular epithelium; many tubules filled with necrotic epithelium and casts; many tubules lined with regenerating epithelium.

Experiment 4.-Rabbit killed on the fourth day after the administration of uranium nitrate.

First day. (Wt. $2,150 \mathrm{gm}$.)

Urine : twenty-four-hour amount .............. 110 c.c.

"d" ................................ 128

absolute diastatic strength $\ldots \ldots \ldots \ldots \ldots \ldots, \quad 7,040$

Blood : serum "d" .......................... 64 non-protein nitrogen (per $100 \mathrm{gm}$.) ......... $30 \mathrm{mg}$

Administered $7 \mathrm{mg}$. uranium nitrate.

Second day. (Wt. 2,050 gm.)

Urine: twenty-four-hour amount $\ldots \ldots \ldots \ldots \ldots \ldots, 100$ c.c.

"d" $\ldots \ldots \ldots \ldots \ldots \ldots \ldots \ldots \ldots \ldots \ldots \ldots \ldots, \quad 128$

absolute diastatic strength ................ 6,400

Third day. (Wt. 2,000 gm.)

Urine: twenty-four-hour amount $\ldots \ldots \ldots \ldots \ldots \ldots . \quad 90$ c.c.

"d" ............................... 128

absolute diastatic strength $\ldots \ldots \ldots \ldots \ldots \ldots, \quad 5,760$

Fourth day. (Wt. $1,930 \mathrm{gm}$.)

Urine: twenty-four-hour amount $\ldots \ldots \ldots \ldots \ldots \ldots, \quad 8$ c.c.

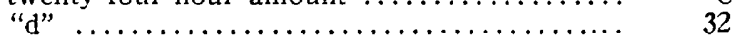

absolute diastatic strength ............. 128

Fifth day. (Wt. 1,950 gm.)

Urine: twenty-four-hour amount ............... Anuric

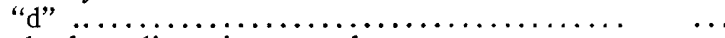

absolute diastatic strength....................

Blood : serum "d" ......................... 64 non-protein nitrogen (per $100 \mathrm{gm}$.) $\ldots \ldots \ldots .196 \mathrm{mg}$.

Histological Findings: Glomeruli negative; considerable necrosis and desquamation of the epithelium of many tubules; many tubules filled with necrotic material, a few with casts; a few tubules lined with regenerating epithelium.

Experiment 5.-Rabbit killed on the ffth day after the administration of the uranium nitrate.

First day. (Wt. 2,050 gm.)

Urine: twenty-four-hour amount $\ldots \ldots \ldots \ldots \ldots \ldots, 180$ c.c. "d"

absolute diastatic strength $\ldots \ldots \ldots \ldots \ldots \ldots, \quad 2,880$

Blood: serum "d" ......................... 32

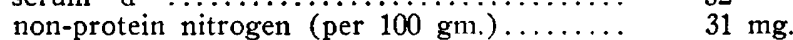

Administered $5 \mathrm{mg}$. uranium nitrate.

Second day. (Wt. 2,000 gm.)

Urine: twenty-four-hour amount ............. 170 c.c.

"d" ..................................... $\quad 32$

absolute diastatic strength $\ldots \ldots \ldots \ldots \ldots \ldots \ldots \ldots, 2,720$ 
Third day. (Wt. 1,900 gm.)

Urine: twenty-four-hour amount $\ldots \ldots \ldots \ldots \ldots \ldots \ldots, \quad 100$ c.c.

absolute diastatic strength ............... 1,600

Fourth day. (Wt. 1,900 gm.)

Urine: twenty-four-hour amount .............. 75 c.c.

"d" $\ldots \ldots \ldots \ldots \ldots \ldots \ldots \ldots \ldots \ldots \ldots \ldots \ldots, \quad 32$

absolute diastatic strength $\ldots \ldots \ldots \ldots \ldots \ldots, 1,200$

Fifth day. (Wt. $1,850 \mathrm{gm}$.)

Urine: twenty-four-hour amount $\ldots \ldots \ldots \ldots \ldots \ldots, \quad 80$ c.c.

"d" .................................. $\quad 32$

absolute diastatic strength ............... 1,280

Sixth day. (Wt. $1,850 \mathrm{gm}$.)

Urine: twenty-four-hour amount .............. 110 c.c.

"d" .............................. 16

absolute diastatic strength ................. $\quad 880$

Blood: serum "d" ....................... 64

$78 \mathrm{mg}$

Histological Findings: Glomeruli negative; considerable necrosis and desquamation of the epithelium of many tubules; a few tubules filled with necrotic material; many tubules lined with regenerating epithelium.

Experiment 6.-Rabbit killed on the sixth day after the administration of the uranium nitrate.

First day. (Wt. $2,100 \mathrm{gm}$.)

Urine: twenty-four-hour amount $\ldots \ldots \ldots \ldots \ldots \ldots$

"d" .....................................

absolute diastatic strength $\ldots \ldots \ldots \ldots \ldots \ldots$.

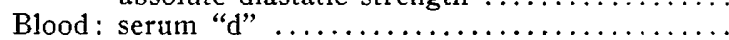
non-protein nitrogen (per $100 \mathrm{gm}$.) ..........

Administered $7 \mathrm{mg}$. uranium nitrate.

Second day. (Wt. 2,100 gm.)

Urine: twenty-four-hour amount

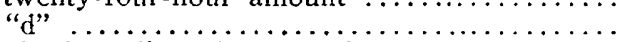

absolute diastatic strength...................

80 c.c.
16
640
64
$35 \mathrm{mg}$.

Third day. (Wt. 2,100 gm.)

Urine: twenty-four-hour amount $\ldots \ldots \ldots \ldots \ldots \ldots$

"d" .....................................

absolute diastatic strength

65 c.c.

64

2,080

100 c.c.

64

3,200

Fourth day. (Wt. 2,000 gm.)

Urine: twenty-four-hour amount $\ldots \ldots \ldots \ldots \ldots \ldots$

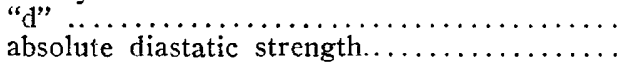

60 c.c.

64

1,920

Fifth day. (Wt. 2,000 gm.)

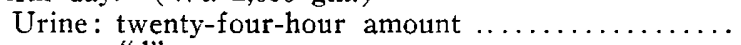

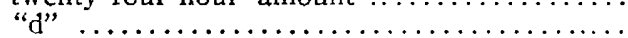

absolute diastatic strength

10 c.c.

4

Sixth day. (Wt. $2,000 \mathrm{gm}$.)

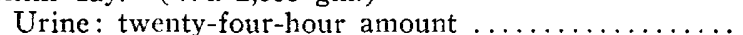

$$
\text { "d" }
$$

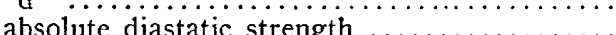

20

20 c.c.

80

Seventh day. (Wt. 2,000 gm.)

Urine: twenty-four-hour amount $\ldots \ldots \ldots \ldots \ldots \ldots$

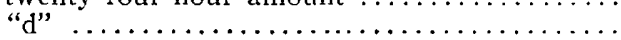

absolute diastatic strength

30 c.c.

4

60

128

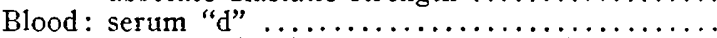

non-protein nitrogen (per $100 \mathrm{gm}$.) $\ldots \ldots \ldots \ldots . .217 \mathrm{mg}$.

Histological findings: Hyaline droplets in many glomeruli; considerable necrosis and desquamation of the epithelium of many tubules; many tubules filled with necrotic material and casts; many tubules lined with regenerating epithelium. 
Experiment 7.-Rabbit killed on the seventh day after the administration of the uranium nitrate.

First day. (Wt. 2,300 gm.)

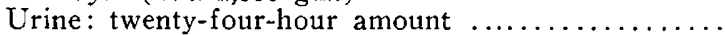

"d"

absolute diastatic strength $\ldots \ldots \ldots \ldots \ldots \ldots$

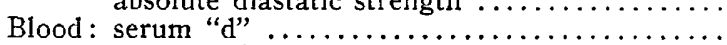
non-protein nitrogen (per $100 \mathrm{gm}$.) ........

Administered $7 \mathrm{mg}$. uranium nitrate.

Second day. (Wt. 2,250 gm.)

Urine: twenty-four-hour amount $\ldots \ldots \ldots \ldots \ldots \ldots$

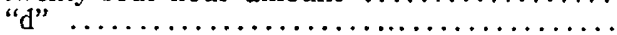

absolute diastatic strength $\ldots \ldots \ldots \ldots \ldots \ldots$

Third day. (Wt. 2,200 gm.)

Urine: twenty-four-hour amount ........... 200 c.c.

"d"

130 c.c.

16

1,040

32

$38 \mathrm{mg}$. absolute diastatic strength

Fourth day: (Wt. 2,150 gm.)

Urine: twenty-four-hour amount

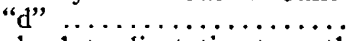

130 c.c.

absolute diastatic strength

Fifth day. (Wt. 1,950 gm.)

Urine: twenty-four-hour amount ............ 70

"d"

absolute diastatic strength $\ldots \ldots \ldots \ldots \ldots \ldots \ldots$

Sixth day. (Wt. 2,000 gm.)

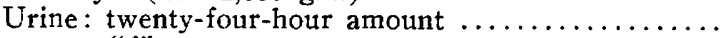

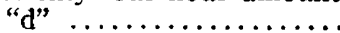

16

560

absolute diastatic strength

100 c.c.

16

800

Seventh day. (Wt. 1,900 gm.)

Urine: twenty-four-hour amount

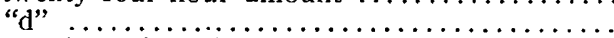

absolute diastatic strength

170 c.c.

12

1,020

Eighth day. (Wt. 1,700 gm.)

Urine: twenty-four-hour amount $\ldots \ldots \ldots \ldots \ldots \ldots$ "d" absolute diastatic strength

120 c.c.

16

960

Blood: Serum "d"

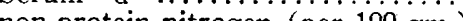

128

$250 \mathrm{mg}$.

Histological Findings: Glomeruli negative; desquamation of the epithelium of a few tubules; many tubules filled with serum and casts; many tubules lined with regenerating epithelium; slight increase in connective tissue throughout the kidney, suggesting preexisting chronic nephritis.

Experiment 8.-Rabbit killed on the eighth day after the administration of the uranium nitrate.

First day. (Wt. 1,500 gm.)

Urine: twenty-four-hour amount ............. 140 c.c.

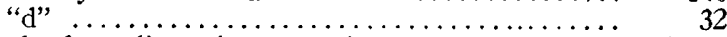

absolute diastatic strength $\ldots \ldots \ldots \ldots \ldots \ldots \ldots, 2,240$

Blood: serum "d" . . .................... 64 non-protein nitrogen (per $100 \mathrm{gm}$.) $\ldots \ldots \ldots \ldots .27 \mathrm{mg}$.

Administered $4 \mathrm{mg}$. uranium nitrate.

Second day. (Wt. $1,450 \mathrm{gm}$.)

Urine : twenty-four-hour amount ............ 150 c.c

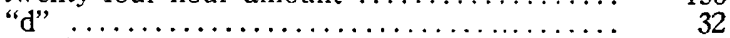

absolute diastatic strength $\ldots \ldots \ldots \ldots \ldots \ldots \ldots, 2,400$

Third day. (Wt. 1,500 gm.)

Urine: twenty-four-hour amount ............ 110 c.c.

"d" .......................... 32

absolute diastatic strength $\ldots \ldots \ldots \ldots \ldots \ldots \ldots, 1,760$ 
Fourth day. (Wt. 1,500 gm.)

Urine: twenty-four-hour amount $\ldots \ldots \ldots \ldots \ldots \ldots$

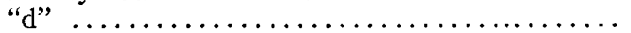
absolute diastatic strength $\ldots \ldots \ldots \ldots \ldots \ldots$ 170 c.c.

Fifth day. (Wt. $1,450 \mathrm{gm}$.)

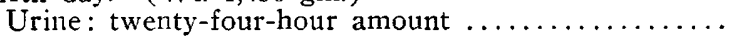

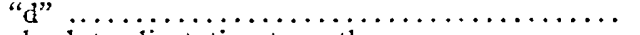

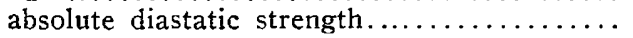

Sixth day. (Wt. 1,450 gm.)

Urine: twenty-four-hour amount $\ldots \ldots \ldots \ldots \ldots \ldots$

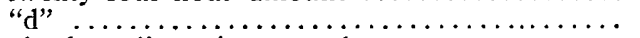
absolute diastatic strength $\ldots \ldots \ldots \ldots \ldots \ldots$

Seventh day. (Wt. 1,400 gm.)

Urine: twenty-four-hour amount

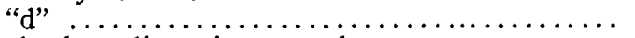
absolute diastatic strength ..............

Eighth day. (Wt. 1,350 gm.)

Urine: twenty-four-hour amount $\ldots \ldots \ldots \ldots \ldots \ldots$

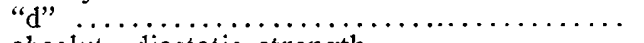
absolute diastatic strength

Ninth day. (Wt. 1,300 gm.)

Urine: twenty-four-hour amount $\ldots \ldots \ldots \ldots \ldots \ldots \ldots$

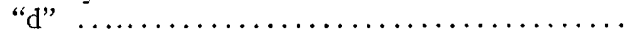
absolute diastatic strength $\ldots \ldots \ldots \ldots \ldots \ldots$

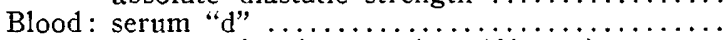
non-protein nitrogen (per $100 \mathrm{gm}$ ).........

75 c.c.

32

1,200

64

$83 \mathrm{mg}$.

Histological Findings: Glomeruli negative; desquamation of the epithelium of a few tubules; many tubules filled with casts and a few with necrotic cells; a few tubules lined with regenerating epithelium.

Experiment 9.-Rabbit killed on the ninth day after the administration of uranium nitrate.

First day. (Wt. 2,000 gm.)

Urine: twenty-four-hour amount $\ldots \ldots \ldots \ldots \ldots \ldots \ldots, 180$ c.c

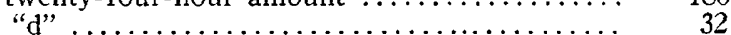

absolute diastatic strength $\ldots \ldots \ldots \ldots \ldots \ldots \ldots \ldots, \quad 2,880$

Blood : serum "d" $\ldots \ldots \ldots \ldots \ldots \ldots \ldots \ldots \ldots \ldots \ldots \ldots \ldots$. 32

$32 \mathrm{mg}$

Administered $4 \mathrm{mg}$. uranium nitrate.

Second day. (Wt. 2,100 gm.)

Urine: twenty-four-hour amount $\ldots \ldots \ldots \ldots \ldots \ldots$

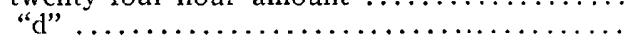

absolute diastatic strength $\ldots \ldots \ldots \ldots \ldots \ldots \ldots$

Third day. (Wt. 2,150 gm.)

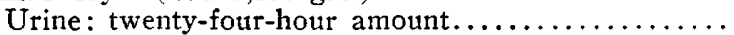

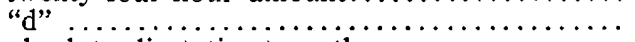

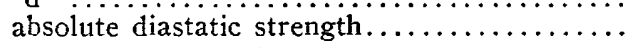

Fourth day. (Wt. 2,100 gm.)

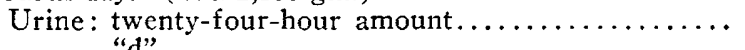

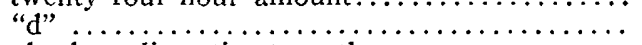

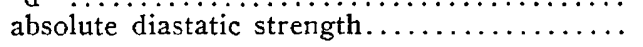

Fifth day. (Wt. 2,100 gm.)*

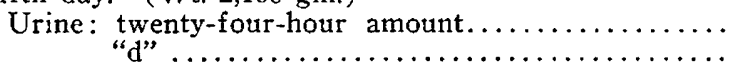

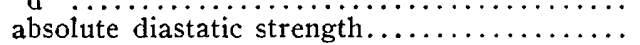

Sixth day. (Wt. 2,050 gm.)

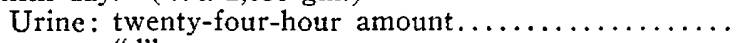

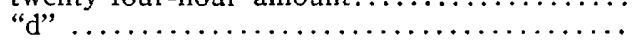

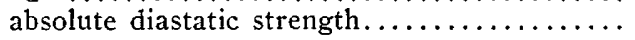
140 c.c.
64
4,680

150 c.c.

32

2,400

230
32

3,680

210 c.c.

3,360

180 c.c.

64

5,760

* On the fifth day the animal received an additional injection of $7 \mathrm{mg}$. of uranium nitrate. 
Seventh day. (Wt. 2,050 gm.)

Urine: twenty-four-hour amount............ 150 c.c.

"d" ........................... 64

absolute diastatic strength............. 4,800

Eighth day. (Wt. 1,950 gm.)

Urine: twenty-four-hour amount............... 100 c.c.

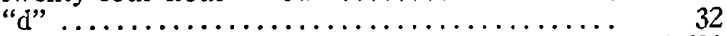

absolute diastatic strength............ 1,600

Ninth day. (Wt. 1,950 gm.)

Urine: twenty-four-hour amount............

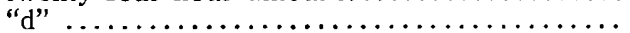

absolute diastatic strength............. 1,440

Tenth day. (Wt. 1,900 gm.)

Urine: twenty-four-hour amount............. 100 c.c.

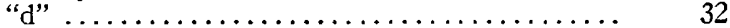

absolute diastatic strength............... 1,600

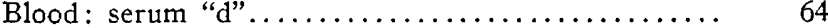

non-protein nitrogen (per $100 \mathrm{gm}$. )........ $96 \mathrm{mg}$

Histological Findings: Glomeruli negative; a few tubules lined with regenerating epithelium; many tubules filled with casts.

Experiment 10.-Rabbit killed on the tenth day after the administration of uranium nitrate.

First day. (Wt. 2,300 gm.)

Urine: twenty-four-hour amount.

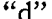

absolute diastatic strength $\ldots \ldots \ldots \ldots \ldots \ldots$

Blood : serum "d" $\ldots \ldots \ldots \ldots \ldots \ldots \ldots \ldots \ldots \ldots \ldots$ non-protein nitrogen (per $100 \mathrm{gm}.) \ldots \ldots \ldots$

Administered $6 \mathrm{mg}$. uranium nitrate.

Second day. (Wt. 2,300 gm.)

Urine: twenty-four-hour amount.

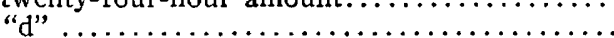

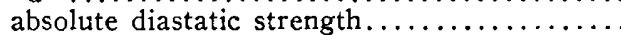

Third day. (Wt. 2,300 gm.)

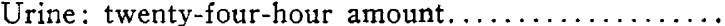

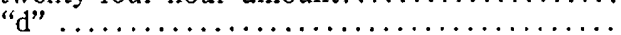

absolute diastatic strength.

180 c.c.

64

5,760

64

$30 \mathrm{mg}$.

120 c.c.

3,840

180 c.c.

64

5,760

Fourth day. (Wt. 2,350 gm.)

Urine: twenty-four-hour amount

"d" absolute diastatic strength

90 c.c.

64

2,850

Fifth day. (Wt. 2,250 gm.)

Urine: twenty-four-hour amount.............

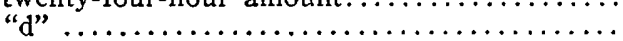

absolute diastatic strength

170 c.c.

32

2,720

Sixth day. (Wt. 2,200 gm.)

Urine: twenty-four-hour amount

"d"

210 c.c. absolute diastatic strength

Seventh day. (Wt. 2,150 gm.)

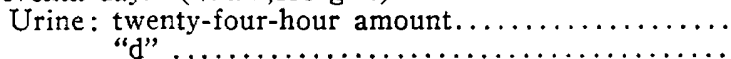

"d" $\ldots \ldots \ldots \ldots \ldots \ldots \ldots \ldots \ldots \ldots \ldots \ldots \ldots \ldots \ldots$

absolute diastatic strength.

3,360

Eighth day. (Wt. 2,150 gm.)

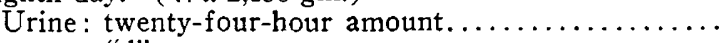

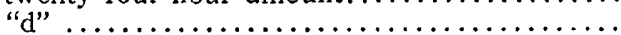

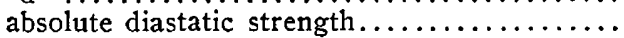

Ninth day. (Wt. 2,150 gm.)

Urine: twenty-four-hour amount

"d"

170 c.c.

64

5,440

220 c.c.

50

5,500

190 c.c

64

absolute diastatic strength

6,080 
Tenth day. (Wt. 2,200 gm.)

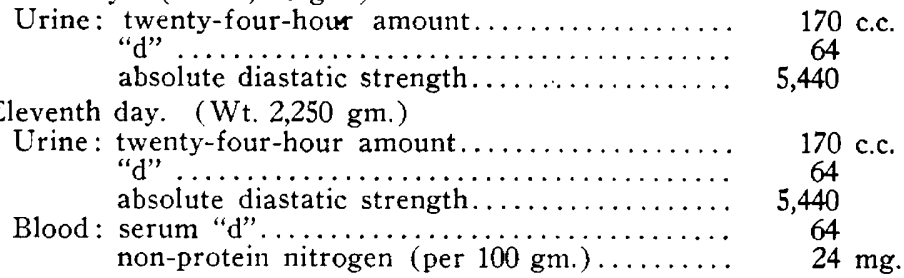

Histological Findings: Glomeruli negative; a few tubules filled with casts.

From Experiments 1 to 10 recorded above, it is seen that the findings in regard to the non-protein nitrogen of the blood confirm those recorded by Frothingham, Fitz, Folin and Denis. ${ }^{15}$ The accumulation of nitrogen was gradual. It became high after the third day, remained at a high level from the third to the eighth day, and then gradually returned to normal. When extensive necrosis of the renal epithelium occurred, the blood nitrogen accumulated rapidly, and diminished slowly with repair of the kidney. Thus this test paralleled in a general way the anatomical damage in the kidney.

The amylase readings in the urine varied considerably in animals supposed to be normal. The "d" readings ran from 16 to 128 units, and the "absolute diastatic strength" from 640 to 5,760 units. This may in part be due to the fact that the animals were not kept under a longer period of preliminary observation. They were taken from stock and injected with uranium nitrate after one twenty-four hour specimen of urine was collected. Nevertheless the blood nitrogen and the blood " $\mathrm{d}$ " readings were much more constant under the same conditions.

In six of the ten experiments there was an increase in the "absolute diastatic strength" of the urine, and in two experiments of the "d" reading, following the injection of the uranium nitrate. This lasted for a short time. It was not caused by an increased output of urine in every case, and suggested a stimulated renal function which was not confirmed, however, by a diminished blood nitrogen.

The "absolute diastatic strength" and the " $\mathrm{d}$ " of the urine diminished in each case as the nephritis became severe. Both readings paralleled roughly the severity of the lesion as judged by anatomical changes in the kidney. The "absolute diastatic strength" seemed to show finer distinctions in regard to the renal function than did the " $\mathrm{d}$ " readings. This was largely because of the variations in the twentyfour hour urine which occurred from day to day.

The blood "d" readings did not increase as much as would be presumed from the variations in the excretion of amylase. In four cases there was a definite rise in " $\mathrm{d}$ " at the end of the experiment when the nephritis was most severe. It did not parallel the accumulation of blood nitrogen, nor accompany an extremely low amylase excretion in 
each case and was not sufficiently marked to make the determinations of particular diagnostic or prognostic value.

Thus on the whole, in the individual cases there was a definite relation between the nephritis, the accumulation of non-protein nitrogen in the blood, and the excretion of amylase in the urine. The blood "d" readings were insignificant.

In order to compare more accurately the relation of the accumulation of the non-protein nitrogen in the blood with the excretion of amylase at different stages of the acute nephritis, a second series of experiments was made. In this series the blood was collected every day from the ear veins and the amylase excretion was followed. Experiments 11 to 19 recorded below, give the important points of these observations.

Experiment 11.-Rabbit killed on the fourth day after the administration of uranium nitrate.

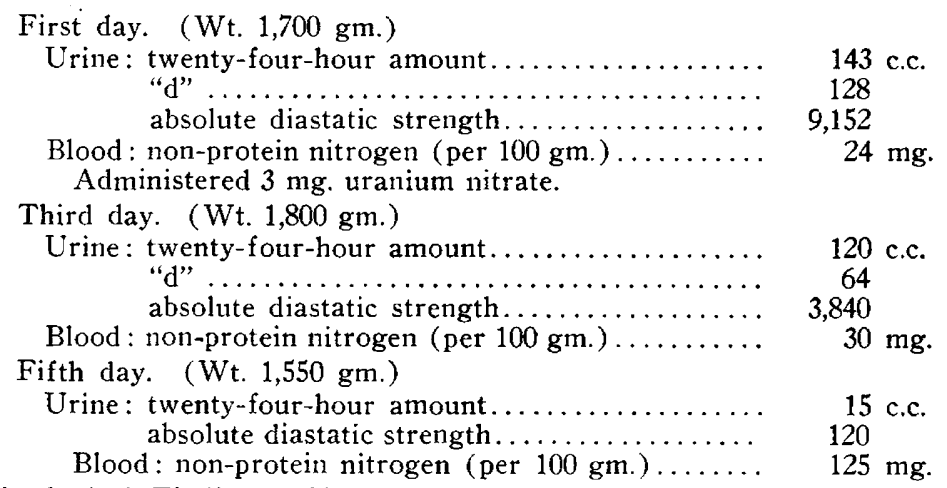

Histological Findings: Glomeruli negative; considerable necrosis and desquamation of the epithelium of certain tubules; many other tubules filled with necrotic material and casts; a few other tubules lined with regenerated epithelium; a few areas of round-cell infiltration.

Experiment 12.-Rabbit found dead on sixth day after the administration of uranium nitrate.

First day. (Wt. $2,500 \mathrm{gm}$.)

Urine: twenty-four-hour amount.............. 105 c.c.

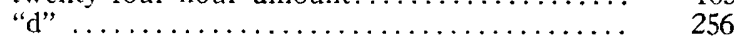

absolute diastatic strength................... 13,440

Blood: non-protein nitrogen (per $100 \mathrm{gm}$.)........ $28 \mathrm{mg}$.

Administered $4 \mathrm{mg}$. uranium nitrate.

Fourth day. (Wt. 2,550 gm.)

Urine: twenty-four-hour amount $\ldots \ldots \ldots \ldots \ldots \ldots \ldots, 135$ c.c.

"d" ................................. 64

absolute diastatic strength................... 4,320

Blood : non-protein nitrogen (per $100 \mathrm{gm}$.)....... $51 \mathrm{mg}$.

Fifth day. (Wt. 2,500 gm.)

Urine: twenty-four-hour amount............... 60 c.c.

"d" $\ldots \ldots \ldots \ldots \ldots \ldots \ldots \ldots \ldots \ldots \ldots \ldots, \quad 128$

absolute diastatic strength............... 3,840

Blood: non-protein nitrogen (per $100 \mathrm{gm}$.)....... $80 \mathrm{mg}$. 
Sixth day. (Wt. 2,500 gm.)

Urine: twenty-four-hour amount $\ldots \ldots \ldots \ldots \ldots \ldots, 10$ c.c.

"d" $\ldots \ldots \ldots \ldots \ldots \ldots \ldots \ldots \ldots \ldots \ldots, 128$

absolute diastatic strength................... 640

Blood: non-protein nitrogen (per $100 \mathrm{gm}$.)........ $125 \mathrm{mg}$.

Histological Findings: Glomeruli congested; considerable necrosis and desquamation of the epithelium of certain tubules; many other tubules filled with necrotic material and casts; a few other tubules lined with regenerated epithelium.

Experiment 13.-Rabbit found dead on sixth day after the administration of uranium nitrate.

First day. (Wt. 2,300 gm.)

Urine: twenty-four-hour amount............. 100 c.c.

"d" ............................ 64

absolute diastatic strength................. 3,200

Blood : non-protein nitrogen (per $100 \mathrm{gm}$.)........ $25 \mathrm{mg}$.

Administered $8 \mathrm{mg}$. uranium nitrate.

Third day. (Wt. 2,350 gm.)

Urine: twenty-four-hour amount.............. 40 c.c.

"d" .............................. 128

absolute diastatic strength................. 2,560

Blood: non-protein nitrogen (per $100 \mathrm{gm}$.)....... $41 \mathrm{mg}$.

Fourth day. (Wt. $2,350 \mathrm{gm}$.)

Urine: twenty-four-hour amount $\ldots \ldots \ldots \ldots \ldots \ldots \ldots, \quad 3$ c.c.

"d" .................................. 32

absolute diastatic strength................... 48

Blood: non-protein nitrogen (per $100 \mathrm{gm}$.)........ $70 \mathrm{mg}$.

Fifth day. (Wt. 2,300 gm.)

Urine: twenty-four-hour amount..............

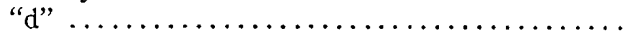

absolute diastatic strength.....................

Blood: non-protein nitrogen (per $100 \mathrm{gm}$.) ...........

Sixth day. (Wt. 2,300 gm.)

Urine: twenty-four-hour amount................ 7 c.c.

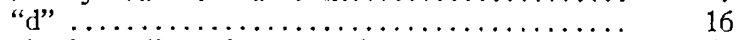

absolute diastatic strength.................. 56

Blood: non-protein nitrogen (per $100 \mathrm{gm}$. )........ $125 \mathrm{mg}$.

Histological Findings: Glomeruli negative: necrosis and desquamation of the epithelium of certain tubules; few tubules filled with necrotic material, but many filled with casts; many tubules lined with regenerated epithelium.

Experiment 14.-Rabbit killed on the seventh day after the administration of uranium nitrate.

First day. (Wt. 2,000 gm.)

Urine: twenty-four-hour amount.............. 120 c.c.

"d" .................................... 128

absolute diastatic strength............... 7,680

Blood: non-protein nitrogen (per $100 \mathrm{gm}$.)....... $30 \mathrm{mg}$.

Administered $2.5 \mathrm{mg}$. uranitum nitrate.

Second day. (Wt. 1,950 gm.)

Urine: twenty-four-hour amount............. 170 c.c.

"d" ............................... 128

absolute diastatic strength............... 10,880

Blood: non-protein nitrogen (per $100 \mathrm{gm}$. )....... $35 \mathrm{mg}$

Fourth day. (Wt. $1,950 \mathrm{gm}$.)

Urine: twenty-four-hour amount.............. 17 c.c.

"d" ....................................... 256

absolute diastatic strength................ 2,176

Blood: non-protein nitrogen (per $100 \mathrm{gm}$.)........ $136 \mathrm{mg}$. 
Sixth day. (Wt. $1,950 \mathrm{gm}$.)

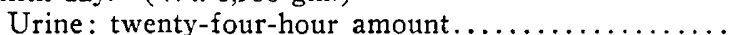

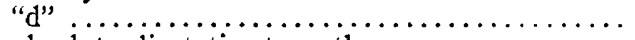

absolute diastatic strength.....................

Blood: non-protein nitrogen (per $100 \mathrm{gm}$.).........

20 c.c.

4

40

$200 \mathrm{mg}$.

Seventh day. (Wt. 1,950 gm.)

Urine: twenty-four-hour amount................

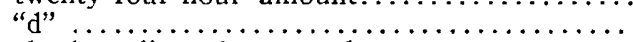

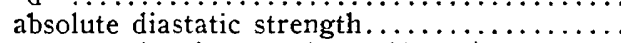

Blood: non-protein nitrogen (per $100 \mathrm{gm}.) \ldots \ldots \ldots$
8 c.c.

8

$238 \mathrm{mg}$.

Histological Findings: Glomeruli negative; considerable necrosis and desquamation of the epithelium of certain tubules; many tubules flled with necrotic material and casts; many tubules lined with regenerated epithelium.

Experiment 15.-Experiment discontinued on the ninth day after the administration of the uranium nitrate.

First day. (Wt. 2,700 gm.)

Urine: twenty-four-hour amount.............. 150 c.c.

"d" ................................... 128

absolute diastatic strength.............. 9,600

Blood: non-protein nitrogen (per $100 \mathrm{gm}$.) ........ $27 \mathrm{mg}$.

Administered $4 \mathrm{mg}$. uranium nitrate.

Third day. (Wt. 2,650 gm.)

Urine: twenty-four-hour amount.............. 200 c.c.

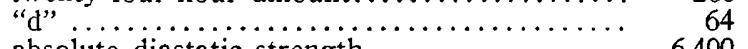

absolute diastatic strength.................. 6,400

Blood : non-protein nitrogen (per $100 \mathrm{gm}$.)....... $37 \mathrm{mg}$.

Fifth day. (Wt. 2,600 gm.)

Urine: twenty-four-hour amount............... 180 c.c.

"d" $\ldots \ldots \ldots \ldots \ldots \ldots \ldots \ldots \ldots \ldots \ldots \ldots \ldots \ldots, \quad 64$

absolute diastatic strength.............. 5,760

Blood : non-protein nitrogen (per $100 \mathrm{gm}$. )..........

Seventh day. (Wt. 2,450 gm.)

Urine: twenty-four-hour amount.............. 100 c.c.

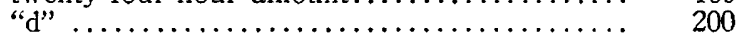

absolute diastatic strength............... 10,000

Blood : non-protein nitrogen (per $100 \mathrm{gm}.) \ldots \ldots \ldots .654 \mathrm{mg}$.

Ninth day. (Wt. 2,350 gm.)

Urine: twenty-four-hour amount.............. 100 c.c.

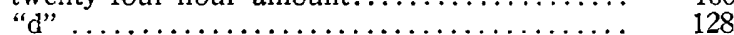

absolute diastatic strength................. 6,400

Blood : non-protein nitrogen (per $100 \mathrm{gm}$.) ........ $43 \mathrm{mg}$.

Experiment 16.-Rabbit killed on the tenth day after the administration of uranium nitrate.

First day. (Wt. 2,700 gm.)

Urine: twenty-four-hour amount............... 220 c.c.

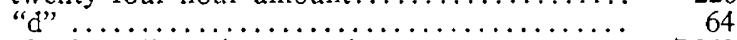

absolute diastatic strength...................... $\quad 7,040$

Blood: non-protein nitrogen (per $100 \mathrm{gm}$.)........ $38 \mathrm{mg}$.

Administered $4 \mathrm{mg}$. uranium nitrate.

Third day. (Wt. 2,700 gm.)

Urine: twenty-four-hour amount............. 220 c.c.

"d" .................................. 128

absolute diastatic strength..................... 14,080

Blood: non-protein nitrogen (per $100 \mathrm{gm}$.)........ $46 \mathrm{mg}$.

Fifth day. (Wt. 2,600 gm.)

Urine: twenty-four-hour amount.............. 50 c.c.

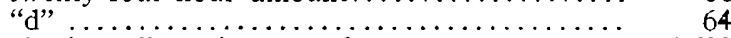

absolute diastatic strength............... 1,600

Blood: non-protein nitrogen (per $100 \mathrm{gm}$.)........ $102 \mathrm{mg}$. 
Sixth day. (Wt. 2,500 gm.)

Urine: twenty-four-hour amount............... 70 c.c.

"d"

absolute diastatic strength $\ldots \ldots \ldots \ldots \ldots \ldots$

Blood : non-protein nitrogen (per $100 \mathrm{gm}$.)........ $161 \mathrm{mg}$.

Eighth day. (Wt. 2,300 gm.)

Urine: twenty-four-hour amount...

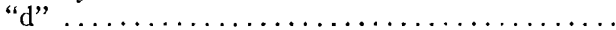

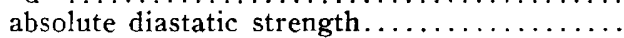

Blood: non-protein nitrogen (per $100 \mathrm{gm}$. ).........

Tenth day. (Wt. 2,100 gm.)

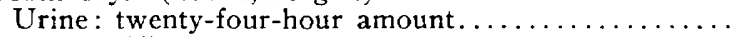
"d"

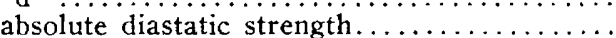

Blood: non-protein nitrogen (per $100 \mathrm{gm}$. ).........

80 c.c.

32

1,280

$208 \mathrm{mg}$.

140 c.c.

64

4,680

$165 \mathrm{mg}$.

Histological Findings: Glomeruli negative; epithelium of tubules negative; many tubules contain hyaline casts.

Experiment 17 .- Rabbit found dead on the tenth day after the administration of the uranium nitrate.

First day. (Wt. 1,900 gm.)

Urine: twenty-four-hour amount............. 100 c.c.

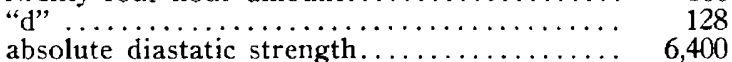

Blood: non-protein nitrogen (per $100 \mathrm{gm}$.)....... $34 \mathrm{mg}$.

Administered $2 \mathrm{mg}$. uranium nitrate.

Third day. (Wt. $2,050 \mathrm{gm}$.)

Urine: twenty-four-hour amount........... 95 c.c.

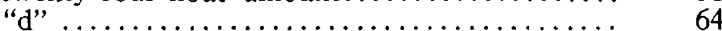

absolute diastatic strength ........... 3,040

Blood: non-protein nitrogen (per $100 \mathrm{gm}$ )........ $41 \mathrm{mg}$.

Fifth day. (Wt. 2,050 gm.)

Urine: twenty-four-hour amount............ 45 c.c.

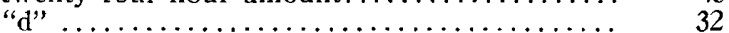

absolute diastatic strength............ 720

Blood : non-protein nitrogen (per $100 \mathrm{gm}$.)........... $113 \mathrm{mg}$

Seventh day. (Wt. $1,900 \mathrm{gm}$.)

Urine: twenty-four-hour amount.............

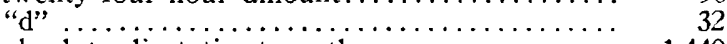

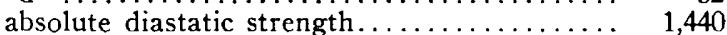

Blood: non-protein nitrogen (per $100 \mathrm{gm}$.)....... $208 \mathrm{mg}$.

Eighth day. (Wt. $1,800 \mathrm{gm}$.)

Urine : twenty-four-hour amount............ 185 c.c.

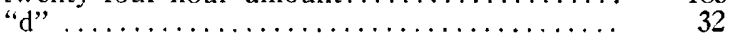

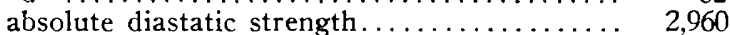

Blood: non-protein nitrogen (per $100 \mathrm{gm}.) \ldots \ldots \ldots \ldots .250 \mathrm{mg}$

Tenth day. (Wt. 1,550 gm.)

Urine: twenty-four-hour amount............. 200 c.c.

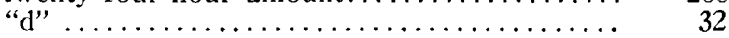

absolute diastatic strength.............. 3,200

Blood : non-protein nitrogen (100 per gm.)....... $151 \mathrm{mg}$.

Histological Findings: Glomeruli negative. A few tubules filled with necrotic material; many tubules filled with hyaline casts; a few tubules lined with regenerating epithelium. A few areas of round-cell infiltration.

Experiment 18.-Experiment discontinued on the thirteenth day after the administration of uranium nitrate.

First day. (Wt. 2,200 gm.)

Urine: twenty-four-hour amount .....................

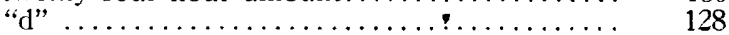

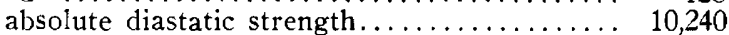

Blood: non-protein nitrogen (per $100 \mathrm{gm}$ )........ $30 \mathrm{mg}$.

Administered $2.5 \mathrm{mg}$. uranium nitrate. 
Second day. (Wt. 2,200 gm.)

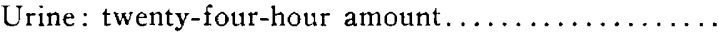

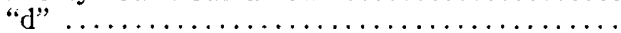
absolute diastatic strength...............

Blood: non-protein nitrogen (per $100 \mathrm{gm}$. ).........

Fourth day. (Wt. 2,100 gm.)

Urine: twenty-four-hour amount...............

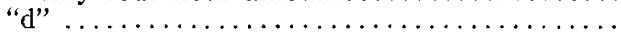

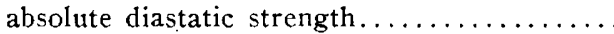

Blood: non-protein nitrogen (per $100 \mathrm{gm}$.)

150 c.c.

128

9,600

$39 \mathrm{mg}$.

125 c.c.

32

2,000

$83 \mathrm{mg}$.

Sixth day. (Wt. 2,000 gm.)

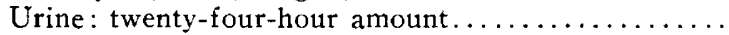

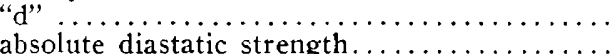

Blood: non-protein nitrogen (per $100 \mathrm{gm}$.).........

Eighth day. (Wt. $1,900 \mathrm{gm}$.)

Urine: twenty-four-hour amount.

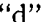

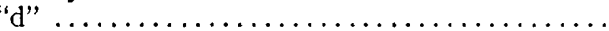

absolute diastatic strength

Blood : non-protein nitrogen (per $100 \mathrm{gm}$.)........

Tenth day. (Wt. $1,950 \mathrm{gm}$.)

Urine: twenty-four-hour amount.

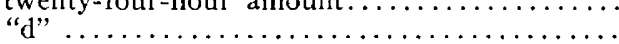

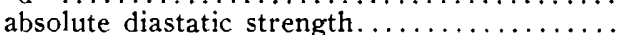

Blood: non-protein nitrogen (per $100 \mathrm{gm}$.) .............

Eleventh day. (Wt. 1,850 gm.)

Urine: twenty-four-hour amount................

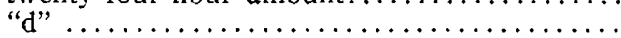
absolute diastatic strength $\ldots \ldots \ldots \ldots \ldots \ldots$

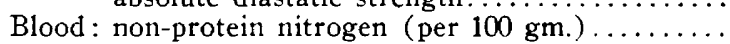

Thirteenth day. (Wt. 2,000 gm.)

Urine: twenty-four-hour amount................

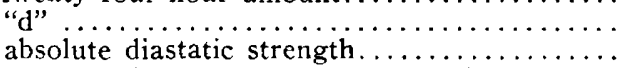

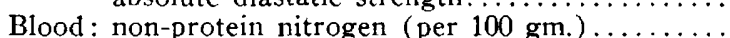

155 c.c.

2,480

$104 \mathrm{mg}$.

170 c.c.

64

$\mathbf{5 , 4 4 0}$

$96 \mathrm{mg}$.

170 c.c.

64

5,440

$83 \mathrm{mg}$.

210 c.c.

64

6,720

$52 \mathrm{mg}$.

110 c.c.

128

7,040

$27 \mathrm{mg}$.

Experiment 19.-Experiment discontinued on the fifteenth day after the injection of uranium nitrate.

First day. (Wt. $2,500 \mathrm{gm}$.)

Urine: twenty-four-hour amount...............

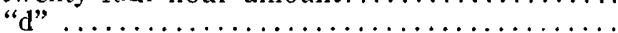

absolute diastatic strength . . . . . . . . . . . .

Blood: non-protein nitrogen (per 100 gm.) .........

Administered $6 \mathrm{mg}$. uranium nitrate.

Second day. (Wt. 2,400 gm.)

Urine: twenty-four-hour amount.

"d"

absolute diastatic strength

200 c.c

100

10,000

$29 \mathrm{mg}$.

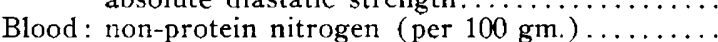

170 c.c.

100

8,500

Fif th day. (Wt. 2,350 gm.)

Urine: twenty-four-hour amount...............

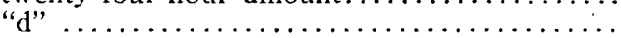

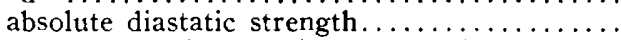

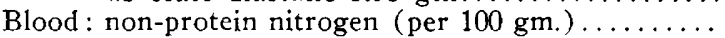

Seventh day. (Wt. 2,400 gm.)

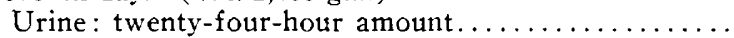

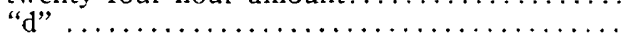

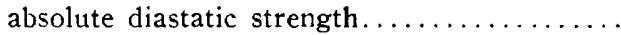

Blood: non-protein nitrogen (per $100 \mathrm{gm}$. ).........

Tenth day. (Wt. 2,100 gm.)

Blood: non-protein nitrogen (per $100 \mathrm{gm}$.)

$43 \mathrm{mg}$.

200 c.c.

12

1,200

$54 \mathrm{mg}$.

150 c.c.

16

1,200

$74 \mathrm{mg}$.

$78 \mathrm{mg}$. 
Thirteenth day. (Wt. 2,200 gm.)

Urine: twenty-four-hour amount.............. 150 c.c.

"d" .................................... 32

absolute diastatic strength................ 2,400

Blood : non-protein nitrogen (per $100 \mathrm{gm}$.)....... $40 \mathrm{mg}$.

Fifteenth day. (Wt. 2,400 gm.)

Urine: twenty-four-hour amount............. 170 c.c.

"d" $\ldots \ldots \ldots \ldots \ldots \ldots \ldots \ldots \ldots \ldots \ldots \ldots \ldots \ldots, 64$

absolute diastatic strength................... $\quad 5,440$

Blood: non-protein nitrogen (per $100 \mathrm{gm}$.)........ $31 \mathrm{mg}$.

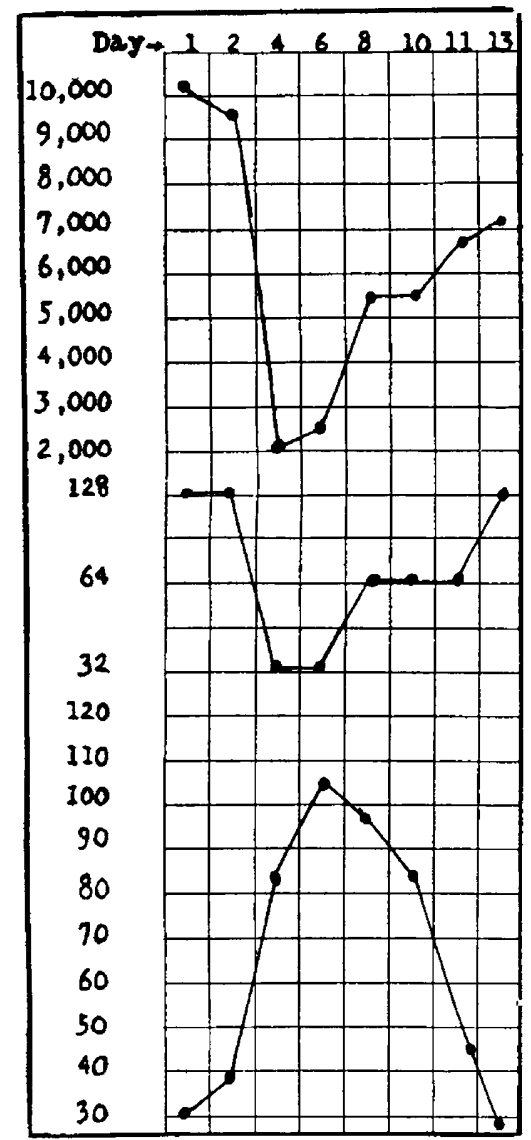

Chart 1.-Curves showing "absolute diastatic" strength (upper curve), "d" excretion (middle curve), and (lower curve) non-protein nitrogen retention in a moderate nephritis (Exp. 18).

The correspondence between the results of the amylase tests and those of the blood analyses in Experiments 11 to 19 is shown graphically in the accompanying charts, (a) with reference to a moderate degree of nephritis (Exp. 18), (b) with reference to severe nephritis (Exp. 17). 
From the tables and charts it is clear that at the beginning of the nephritis the "absolute diastatic strength" of the urine dropped more rapidly than the accumulation of the non-protein nitrogen of the blood. During the course of the disease the height of the nitrogenous accumu-

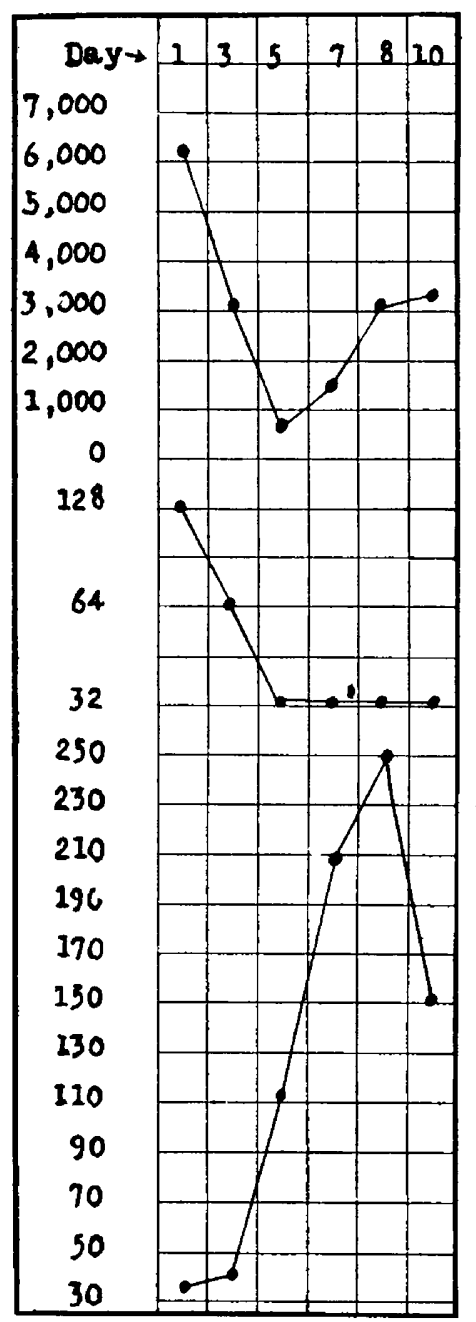

Chart 2.-Curves showing "absolute diastatic" strength (upper curve), "d" excretion (middle curve), and (lower curve) non-protein nitrogen retention in a severe nephritis (Exp. 17).

lation was reached later than the lowest level of the amylase excretion. After the kidney function had begun to improve as shown by an increasing elimination of amylase, the retention nitrogen did not at once begin to recede but even continued to rise for a short period. 
Thus the amylase test is very similar to the phenolsulphonephthalein test. It has certain disadvantages. In a series of nineteen rabbits, the normal excretion showed such marked variation that the information obtained from the test could only be applied to the individual case. Therefore in studying the renal function of any one it was first necessary to discover the normal excretion for that animal. It is only fair to state, however, that the results obtained in man by other observers tend to show that there is much less normal individual variation than in the experiments reported here. A second disadvantage with the test is that it is less quantitative than the phenolsulphonephthalein test. If the "absolute diastatic strength" is taken, small changes in the output of urine may exaggerate changes in renal function. If the " $d$ " reading is accepted, slight changes in renal function are overlooked. Possibly a different method of diluting the urine, as suggested by Brown and Smith, ${ }^{21}$ may obviate this source of error.

On the whole, these experiments show that there is a close parallelism between the modes of excretion of phenolsulphonephthalein and amylase. and that amylase like phenolsulphonephthalein diminishes with an increasing nephritis, returns to normal with improvement of renal function, and is not influenced by blood retention to the same extent as is the accumulation of non-protein nitrogen.

\section{CONCLUSIONS}

1. In acute uranium nephritis in rabbits, the excretion of amylase in the urine and the amount of non-protein nitrogen in the blood vary from the normal during the course of the nephritis and return to normal as the nephritis heals.

2. In individual cases the degree of variation from the normal agrees on the whole with the amount of destruction demonstrated histologically in the kidney.

3. The amylase excretion, like that of phenolsulphonephthalein, drops rapidly to its lowest point and returns rapidly toward its previous level with recovery of the kidney. It is but little influenced by accumulation in the blood.

4. The non-protein nitrogen accumulates gradually in the blood, and returns to normal gradually as the kidney recovers.

5. Amylase excretion as a test for renal function is similar to phenolsulphonephthalein, but is less delicate.

6. The amylase and phenolsulphonephthalein tests show the renal function at the moment; the blood nitrogen test is more influenced by the duration of the condition causing its accumulation.

Peter Bent Brigham Hospital.

21. Brown and Smith: Bull. Johns Hopkins Hosp., 1914, xxv, 213. 